\title{
Duodenitis in Systemic Autoimmune Diseases: Pathologist Perspective
}

\author{
Priyavadhana Balasubramanian'1, Bhawana Ashok Badhe ${ }^{1}$, Rajesh Nachiappa Ganesh ${ }^{1 *}$, \\ Lakshmi C. Panicker ${ }^{2}$, Pazhanivel Mohan²
}

${ }^{1}$ Departments of Pathology, JIPMER, Puducherry, India

${ }^{2}$ Medical Gastroenterology, JIPMER, Puducherry, India

Email: *drngrajesh@gmail.com

How to cite this paper: Balasubramanian, P., Badhe, B.A., Ganesh, R.N., Panicker, L.C. and Mohan, P. (2018) Duodenitis in Systemic Autoimmune Diseases: Pathologist Perspective. Open Journal of Rheumatology and Autoimmune Diseases, 8, 79-86. https://doi.org/10.4236/ojra.2018.83008

Received: June 14, 2018

Accepted: August 3, 2018

Published: August 6, 2018

Copyright (c) 2018 by authors and Scientific Research Publishing Inc. This work is licensed under the Creative Commons Attribution International License (CC BY 4.0).

http://creativecommons.org/licenses/by/4.0/

\begin{abstract}
Aims: To study the histomorphology of duodenitis associated with systemic autoimmune diseases with clinicopathologic correlation. Patients and Methods: This is a descriptive prospective study. Fifteen patients of autoimmune diseases with duodenitis were included. Informed consent was taken. Histomorphological parameters studied were villous architecture, crypt architecture, intraepithelial lymphocyte (IEL) count per 100 enterocytes, villous tip IEL count per 20 enterocytes were counted, inflammatory cells in lamina propria-lymphocytes, neutrophils, eosinophils, epithelioid cells. Statistical analysis was done using IBM-SPSS software version 21. Results: Fifteen cases of duodenitis associated with autoimmune diseases included 6 patients of systemic lupus erythematosus (SLE), 5 of rheumatoid arthritis, one each of ankylosing spondylitis, systemic sclerosis, dermatomyositis and seronegative reactive arthritis. All these cases were serologically proven. Only $3(20 \%)$ patients had mild villous blunting. Six patients (46.7\%) had increased IEL counts. The range of IELs was $8-30$, mean \pm SD was $14 \pm 7.6$. Range of villous tip IELs was $0-8$ with mean \pm SD of $3.45 \pm 2.56$. Six patients (46.7\%) had increased IEL counts but only 3 patients (20\%) had increased villous tip IELs. All patients had moderate increase in lymphoplasmacytic infiltrate in lamina propria. Eosinophils in lamina propria were increased in $46.7 \%$ cases. Conclusion: One of the causes for malabsorptive conditions in adult population in South India is found to be duodenitis associated with autoimmune conditions. We conclude that a combination of clinical, serological, endoscopic and histopathologic features is crucial in arriving at a correct diagnosis.
\end{abstract}

\section{Keywords}

Systemic Autoimmune Diseases, Duodenitis 


\section{Introduction}

Autoimmune disorders comprise a group of immunologic disorders which are related to alterations in the immune system. They include Systemic lupus erythematosus, Rheumatoid arthritis, Scleroderma, Behcet syndrome, Polyarteritis nodosa, Hashimoto thyroiditis, Graves disease, Psoriasis, Multiple sclerosis. The underlying pathology is most commonly due to deposition of immune complexes in blood vessel walls, resulting in ischemia or thrombosis. They are characterised by multisystem involvement, affecting dermatologic, immunologic musculoskeletal, genitourinary, gastrointestinal, cardiovascular and respiratory systems [1]. The gastrointestinal manifestations of these disorders are due to the underlying autoimmune process and shows wide variations. They can either be initial manifestations, or complications of treatment. They can be oral ulcers, gastroesophageal refux disease, abdominal pain, constipation, diarrhoea, fecal incontinence, perforation, gastrointestinal bleeding [2].

Autoimmune diseases can also present with malabsorptive symptoms, with features of duodenitis and increased intraepithelial lymphocyte (IEL) counts, mimicking latent celiac disease. Systemic lupus erythematosus, Rheumatoid arthritis, Hashimoto thyroiditis, Graves disease, Psoriasis and Multiple sclerosis are the most common autoimmune diseases, frequently associated with duodenitis [3]. The most common causes of malabsorption in the developing countries are tropical sprue (TS), parasitic infestations, intestinal tuberculosis while in the West, the commoner causes include celiac disease (CD), crohns disease etc. [4].

The objective of this paper was to study the histomorphology of duodenitis associated with systemic autoimmune diseases with clinicopathologic correlation.

\section{Patients and Methods}

This study was done in the departments of medical gastroenterology and pathology. Out of 101 patients who presented with symptoms of malabsorption, 15 patients had autoimmune diseases associated with duodenitis. We studied the histomorphology of these patients duodenal mucosal biopsies. Histopathological examination of these patients duodenal biopsies was done by standard processing \& staining procedures. Clinicopathological details were collected and informed consent was taken from the patients.

Histomorphological parameters studied were villous architecture, crypt architecture, intraepithelial lymphocyte (IEL) count per 100 enterocytes (average of 300 enterocytes were taken), villous tip IEL count per 20 enterocytes were counted (average of five villi taken), inflammatory cells in lamina propria-lymphocytes, neutrophils, eosinophils, epithelioid cells and its severity was graded as mild, moderate and severe. Interpretation of IEL count and villous tip IEL count was done according to Datta Gupta et al. [5]. Eosinophils were counted per five high power field and less than 22 was taken as normal and more than 22 as increased [6]. 
Statistical analysis was done using IBM-SPSS software version 21. All statistical tests were carried out at $5 \%$ level of significance and $\mathrm{P}$ value $<0.05$ was considered as statistically significant.

\section{Results}

We had 15 cases of duodenitis associated with autoimmune diseases, constituting $15.9 \%$ of the total. We had 6 patients of systemic lupus erythematosus (SLE), 5 of rheumatoid arthritis, one each of ankylosing spondylitis, systemic sclerosis, dermatomyositis and seronegative reactive arthritis. All these cases were serologically proven.

The age of the patients ranged from 19 to 57 years with mean age of $37 \pm$ 10.56 years. There was a female preponderance and diarrhoea was the most common symptom. The mean value of haemoglobin in autoimmune duodenitis was $11.59 \pm 1.54$. Only one case had scalloped mucosal folds. Only 3 (20\%) patients had mild villous blunting. Six patients (46.7\%) had increased IEL counts. The range of IELs was 8 - 30 with mean \pm SD of $14 \pm 7.6$. Range of villous tip IELs was $0-8$ with mean \pm SD of $3.45 \pm 2.56$. Six patients $(46.7 \%)$ had increased IEL counts but only 3 patients (20\%) had increased villous tip IELs. All patients had moderate increase in lymphoplasmacytic infiltrate in lamina propria. Eosinophils in lamina propria was increased in $46.7 \%$ cases. Range of eosinophils was 2 - 130 and mean \pm SD was $30.5 \pm 28.6$. Figure 1 shows (a) Villi showing increased IELs (H\&E, 400×) (b) Increased mixed inflammatory infiltrate comprised of lymphocytes, plasma cells, eosinophils in lamina propria (H\& E, 400×) (c) Increased eosinophils in lamina propria (H\&E, 400×).

Duodenitis associated with autoimmune diseases was compared with non-autoimmune conditions and non-specific duodenitis for demographic parameters, clinical, endoscopic and histologic parameters. There was no statistically significant difference in any of the parameters. But we observed that $93.3 \%$ of autoimmune duodenitis had moderate increase in lymphocytes and plasma cells in lamina propria as compared with other conditions.

\section{Discussion}

Gastrointestinal manifestations are common in primary extraintestinal diseases and systemic diseases. Autoimmune disorders show increased IELs counts with variable villous atrophy. The conditions reported were Systemic lupus erythematosus, Rheumatoid arthritis, Hashimoto thyroiditis, Graves disease, Psoriasis and Multiple sclerosis [3].

In a study done by Kakar et al., 14\% of CD patients had an associated autoimmune condition [7]. In the study done by Brown et al, CD was seen in 2 patients of ankylosing spondylitis and 1 patient of progressive systemic sclerosis [8]. Kakar et al. in his series reported $14 \%$ of autoimmune conditions with increased IELs and in our study $46.7 \%$ patients had increased IEL counts. The patients included in their study were SLE, RA, Psoriasis, Hashimotos thyroiditis, 


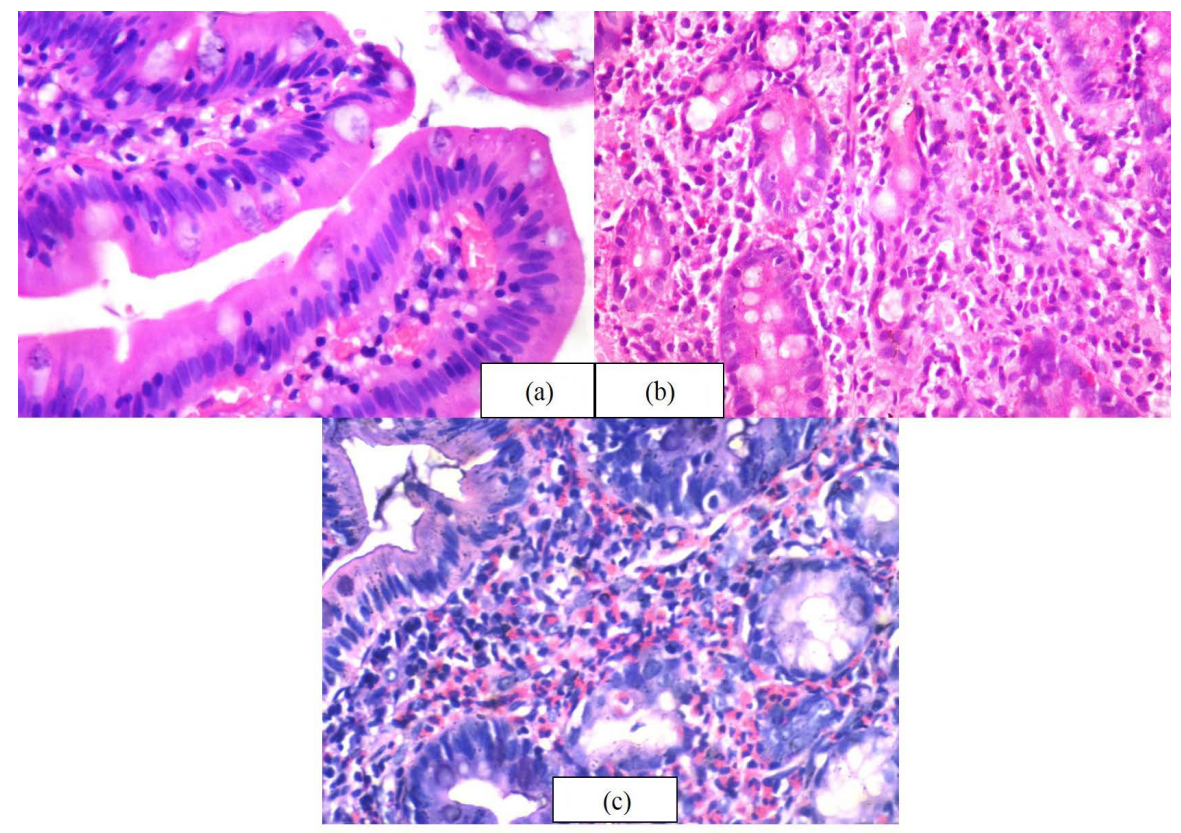

Figure 1. Shows (a) Villi showing increased IELs (H\&E, 400×); (b) Increased mixed inflammatory infiltrate comprised of lymphocytes, plasma cells, eosinophils in lamina propria (H\& E, 400×); (c) Increased eosinophils in lamina propria (H\&E, 400×).

multiple sclerosis, Graves, Ankylosing spondylitis and progressive systemic sclerosis which is similar to the spectrum of autoimmune conditions in our study [7].

SLE is a multisystem autoimmune disease with immunologic abnormalities, autoantibodies to nuclear and cytoplasmic antigens, associated with tissue damage. SLE shows a female predominance, affecting woman in their childbearing year. It can affect many organ systems at different times over the course of the disease. Gastrointestinal manifestations occur in about $25 \%$ to $40 \%$ of cases. They are primarily related to vasculitis or to complications of therapy [1] [9]. The most common GI manifestations are abdominal pain with peritoneal signs, penetrating ulcers and transmural infarction [10] [11]. Also, endoscopic mucosal biopsies usually do not contain the vasculitic lesions. Functional disorders such as malabsorption or protein-losing enteropathy are presumed to be due to ischemia or other mechanisms but not clearly understood [12] [13]. When malabsorption is found in a patient with SLE, other known causes of malabsorption, such as CD should be ruled out. Peptic ulcer disease is common in patients treated with NSAIDs and corticosteroids [14].

The older literature reports the incidence of GI manifestations in SLE patients varying from $1.3 \%$ to $27.5 \%$. In our study, $40 \%$ cases of autoimmune associated duodenitis are due to SLE. Studies have described gastric ulcer in SLE patients but there are no studies describing duodenitis in SLE patients. All the SLE patients statisfied the criteria as per American College of Rheumatology (ACR) [15]. One of our patients had SLE and NSAID induced duodenitis. In two of our SLE patients, one had hookworm, while another patient had fatal Strongyloides 
stercoralis infection possibly due to steroid induced immunosuppression. So it is very important, to have a high index of suspicion for infections in patients on steroids or other immunosuppressive therapy to avoid delay in appropriate treatment.

Few case reports describe biopsy features of villous blunting similar to that seen in CD patients. Worrall et al. and Rustgi et al. in their studies observed that the genetic haplotypes of CD can be seen in SLE patients also [16] [17]. Celiac disease has occurred in 14 case reports simultaneously with lupus and a possible link has been established between the common haplotypes HLA DR3 and HLA B8 seen in both diseases [18] [19]. In our study, 2 SLE patients, who satisfied the ACR criteria were subsequently diagnosed to have celiac disease. One of this patient was incidentally found to be HLA-DQ2 positive which warranted biopsy.

The importance of identifying histologic features of CD in other autoimmune diseases, maybe because of the following reasons like CD is proposed to have an autoimmune etiology. Patients with other autoimmune diseases may be under immunosuppressive management and this may result in subclinical manifestation of $\mathrm{CD}$ by clinical, serological and histopathological findings. In view of overlap of clinical and serological findings in CD and other autoimmune diseases, close observation of endoscopic findings for scalloped margins and biopsy findings of villous blunting and increased IELs is crucial in the differential diagnosis [19].

In this context in our study, mild villous blunting was seen in one case of autoimmune duodenitis, however the IEL counts were normal while all the patients of CD had moderate to severe villous blunting. IELs were raised $(>25 / 100$ enterocytes) in 6 patients of autoimmune duodenitis. Villous tip IELs were raised in 3 patients of autoimmune duodenitis. Villous blunting with increased IEL counts was not seen in any case. It is necessary to do endoscopic duodenal biopsies to screen for latent CD.

The reported incidence of GI manifestations directly related to SLE varies widely in previous studies [20] [21] [22] [23] [24]. Table 1 shows the incidence of gastrointestinal manifestations of SLE in various studies.

Rheumatoid arthritis (RA) is an immune-mediated disease affecting small and large joints symmetrically. Extraarticular manifestations is due to systemic inflammatory response. Many patients with RA have gastrointestinal symptoms, mostly due to drug therapy, especially NSAIDs [25]. Fifty percent or more of patients on these drugs have gastroduodenal lesions [1]. Lymphonodular hyperplasia and increased intraepithelial lymphocytes have been seen in patients with juvenile idiopathic arthritis.

Dermatomyositis and polymyositis are rare connective tissue disorders characterized by symmetric proximal muscle weakness, elevated muscle enzymes, and an inflammatory myopathy. Gastrointestinal symptoms can be seen. Histologic findings included ulcers and erosions, severe acute and chronic inflammation and prominent mucosal and submucosal telangiectasia, but not vasculitis [26] [27]. 
Table 1. Incidence of gastrointestinal manifestations of SLE in various studies.

\begin{tabular}{ccccc}
\hline Sl.No & Studies & Year & $\begin{array}{c}\text { Total No. of cases } \\
\text { with SLE }\end{array}$ & GI manifestations in \% \\
\hline 1 & Couris et al. [20] & 1964 & 231 & 1.3 \\
2 & Estes \& Christain [21] & 1971 & 150 & 16 \\
3 & Matalo \& Albo [22] & 1971 & 51 & 27.5 \\
4 & Shapeero et al. [23] & 1974 & 141 & 14 \\
5 & Zizic et al. [24] & 1978 & 140 & 8 \\
\hline
\end{tabular}

\section{Conclusion}

One of the causes for malabsorptive conditions in adult population in South India was found to be duodenitis associated with autoimmune conditions. We studied 15 patients of autoimmune diseases with duodenitis and concluded that a combination of clinical, serological, endoscopic and histopathologic features was crucial in arriving at a correct diagnosis. The limitation of our study was less number of patients. Further larger studies are recommended to validate this finding.

\section{Conflicts of Interest}

The authors declare no conflicts of interest regarding the publication of this paper.

\section{References}

[1] Schneider, A., Merikhi, A. and Frank, B.B. (2006) Autoimmune Disorders: Gastrointestinal Manifestations and Endoscopic Findings. Gastrointestinal Endoscopy Clinics of North America, 16, 133-151. https://doi.org/10.1016/j.giec.2006.01.013

[2] Cojocaru, M., Cojocaru, I.M., Silosi, I. and Vrabie, C.D. (2011) Gastrointestinal Manifestations in Systemic Autoimmune Diseases. Maedica (Buchar), 6, 45-51.

[3] Casey, M.B., Tazelaar, H.D., Myers, J.L., Hunninghake, G.W., Kakar, S., Kalra, S.X., Ashton, R. and Colby, T.V. (2003) Noninfectious Lung Pathology in Patients with Crohn's Disease. American Journal of Surgical Pathology, 27, 213-219. https://doi.org/10.1097/00000478-200302000-00010

[4] Yadav, P., Das, P., Mirdha, B.R., Gupta, S.D., Bhatnagar, S., Pandey, R.M. and Makharia, G.K. (2011) Current Spectrum of Malabsorption Syndrome in Adults in India. Indian Journal of Gastroenterology, 30, 22-28. https://doi.org/10.1007/s12664-011-0081-0

[5] Datta Gupta, S. (2013) Pathology of Celiac Disease: A Brief Review. Tropical Gastroenterology, 34, 207-226. https://doi.org/10.7869/tg.138

[6] Walker, M.M., Salehian, S.S., Murray, C.E., Rajendran, A., Hoare, J.M., Negus, R., Powell, N. and Talley, N.J. (2010) Implications of Eosinophilia in the Normal Duodenal Biopsy-An Association with Allergy and Functional Dyspepsia. Alimentary Pharmacology and Therapeutics, 31, 1229-1236. https://doi.org/10.1111/j.1365-2036.2010.04282.x

[7] Kakar, S., Nehra, V., Murray, J.A., Dayharsh, G.A. and Burgart, L.J. (2003) Significance of Intraepithelial Lymphocytosis in Small Bowel Biopsy Samples with Normal 
Mucosal Architecture. American Journal of Gastroenterology, 98, 2027-2033. https://doi.org/10.1111/j.1572-0241.2003.07631.x

[8] Brown, I., Mino-Kenudson, M., Deshpande, V. and Lauwers, G.Y. (2006) Intraepithelial Lymphocytosis in Architecturally Preserved Proximal Small Intestinal $\mathrm{Mu}$ cosa. An Increasing Diagnostic Problem with a Wide Differential Diagnosis. Ar chives of Pathology and Lab Medicine, 130, 1020-1025.

[9] Sultan, S.M., Ioannou, Y. and Isenberg, D.A. (1993) A Review of Gastrointestinal Manifestations of Systemic Lupus Erythematosus. Rheumatology (Oxford), 38, 917-932. https://doi.org/10.1093/rheumatology/38.10.917

[10] Andoh, A., Fujiyama, Y., Kitamura, S., Ihara, T., Ueda, K., Miyagawa, A., Hodohara, K., Inoue, H. and Bamba, T. (1997) Acute Lupus Peritonitis Successfully Treated with Steroid Pulse Therapy. Journal of Gastroenterology, 32, 654-657. https://doi.org/10.1007/BF02934116

[11] Papa, M.Z., Shiloni, E. and McDonald, H.D. (1986) Total Colonicnecrosis. A Catastrophic Complication of Systemic Lupuserythematosus. Diseases of the Colon and Rectum, 29, 576-578. https://doi.org/10.1007/BF02554260

[12] Kashihara, T., Fujimori, E., Oki, A., Itoh, T., Hashimoto, K., Kotani, K., Fukuda, H., Tako, H., Kawakami, F., Okuno, G., et al. (1992) Protein-Losing Enteropathy and Pancreatic Involvement in a Case of Connective Tissue Disease. GastroenterologicaJaponica, 27, 246-251. https://doi.org/10.1007/BF02777730

[13] Mader, R., Adawi, M. and Schonfeld, S. (1997) Malabsorption in Systemic Lupus Erythematosus. Clinical and Experimental Rheumatology, 15, 659-661.

[14] Komatireddy, G.R., Marshall, J.B., Aqel, R., Spollen, L.E. and Sharp, G.C. (1995) Association of Systemic Lupus Erythematosus and Gluten Enteropathy. Southern Medical Journal, 88, 673-676. https://doi.org/10.1097/00007611-199506000-00018

[15] Hochberg, M.C. (1997) Updating the American College of Rheumatology Revised Criteria for the Classification of Systemic Lupus Erythematosus. Arthritis and Rheumatology, 40, 1725. https://doi.org/10.1002/art.1780400928

[16] Worrall, J.G., Snaith, M.L., Batchelor, J.R. and Isenberg, D.A. (1990) SLE: A Rheumatological View. Analysis of the Clinical Features, Serology and Immunogenetics of 100 SLE Patients during Long-Term Follow-Up. Quarterly Journal of Medicine, 74, 319-330.

[17] Rustgi, A.K. and Peppercorn, M.A. (1988) Gluten-Sensitive Enteropathy and Systemic Lupus Erythematosus. Archives of Internal Medicine, 148, 1583-1584. https://doi.org/10.1001/archinte.1988.00380070079019

[18] Mirza, N., Bonilla, E. and Phillips, P.E. (2007) Celiac Disease in a Patient with Systemic Lupus Erythematosus: A Case Report and Review of Literature. Clinical Rheumatology, 26, 827-828. https://doi.org/10.1007/s10067-006-0344-9

[19] Balasubramanian, P., Badhe, B.A., Ganesh, R.N., Panicker, L.C. and Mohan, P. (2017) Morphologic Spectrum of Duodenal Biopsies in Malabsorption: A Study from Southern India. Journal of Clinical and Diagnostic Research, 11, 17-21.

[20] Couris, G.D., Block, M.A. and Rupe, C.E. (1964) Gastrointestinal Complications of Collagen Diseases: Surgical Implications. Archives of Surgery, 89, 695-700. https://doi.org/10.1001/archsurg.1964.01320040111019

[21] Estes, D. and Christian, C.L. (1971) The Natural History of Systemic Lupus Erythematosus by Prospective Analysis. Medicine (Baltimore), 50, 85-95. https://doi.org/10.1097/00005792-197103000-00001

[22] Matolo, N.M. and Albo, D. (1971) Gastrointestinal Complications of Collagen Vascular Diseases. Surgical Implications. American Journal of Surgery, 122, 678-682. 
https://doi.org/10.1016/0002-9610(71)90299-6

[23] Shapeero, L.G., Myers, A., Oberkircher, P.E. and Miller, W.T. (1974) Acute Reversible Lupus Vasculitis of the Gastrointestinal Tract. Radiology, 112, 569-574. https://doi.org/10.1148/112.3.569

[24] Zizic, T.M., Stevens, M.B. and Glassen, J.N. (1978) Abdominal Syndromes in SLE and Polyarteritis Nodosa: Predisposing Factors. Arthritis and Rheumatology, 21, 606.

[25] Voutilainen, M., Sokka, T., Juhola, M., Farkkila, M. and Hannonen, P. (1998) Nonsteroidal Anti-Inflammatory Drug Associated Upper Gastrointestinal Lesions in Rheumatoid Arthritis Patients. Relationships to Gastric Histology, Helicobacter pylori Infection, and Other Risk Factors for Peptic Ulcer. Scandinavian Journal of Gastroenterology, 33, 811-816. https://doi.org/10.1080/00365529850171459

[26] Eshraghi, N., Farahmand, M., Maerz, L.L., Campbell, S.M., Deveney, C.W. and Sheppard, B.C. (1998) Adult-Onset Dermatomyositis with Severe Gastrointestinal Manifestations: Case Report and Review of the Literature. Surgery, 123, 356-358. https://doi.org/10.1016/S0039-6060(98)70191-6

[27] Kleckner, F.S. (1970) Dermatomyositis and Its Manifestations in the Gastrointestinal Tract. American Journal of Gastroenterology, 53, 141-146. 